\title{
Analysis of Numerical Measure and Numerical Integration Based on Measure
}

\author{
Yinkun Wang, ${ }^{1,2}$ Jianshu Luo, ${ }^{1}$ and Xiangling Chen ${ }^{3}$ \\ ${ }^{1}$ Department of Mathematics and System Science, National University of Defense Technology, Changsha 410073, China \\ ${ }^{2}$ Department of Mathematics, Syracuse University, Syracuse, NY 13244, USA \\ ${ }^{3}$ College of Mathematics and Computer Science, Hunan Normal University, Changsha 410081, China
}

Correspondence should be addressed to Yinkun Wang; yinkun5522@163.com

Received 12 October 2013; Accepted 19 November 2013

Academic Editor: Abdelghani Bellouquid

Copyright (C) 2013 Yinkun Wang et al. This is an open access article distributed under the Creative Commons Attribution License, which permits unrestricted use, distribution, and reproduction in any medium, provided the original work is properly cited.

We present a convergence analysis for a general numerical method to estimate measure function. By combining Lagrange interpolation, we propose a specific method for approximating the measure function and analyze the convergence order. Further, we analyze the error bound of numerical measure integration and prove that the numerical measure integration can decrease the singularity for singular integrals. Numerical examples are presented to confirm the theoretical results.

\section{Introduction}

A numerical integration based on the definition of the Lebesgue integral was proposed in the paper "A New Approach to Numerical Integration" [1]. The method was said to be particularly useful for integrands which are highly oscillatory in character or singular. It provides an exact reduction of multidimensional integrals to one-dimensional integrals. It appeared to be a more economical way of treating certain multidimensional integrals. For concise writing, we call the new numerical integration proposed by B. L. Burrows the numerical measure integration (NMI).

Consider the integral $\int_{I} f(x) d x$, where $f(x) \in L(I)$ is a nonnegative function and $I=[a, b]$. Firstly, define $E(f(x) \geqslant$ $y):=\{x \in I: f(x) \geqslant y\}$ and $\mu_{f}(y):=m(E(f(x) \geqslant y)), y \in$ $\mathbb{R}$. It is easy to obtain that $\mu_{f}(y)$ is a monotonically decreasing and bounded function with $0 \leqslant \mu_{f}(y) \leqslant b-a, y \in \mathbb{R}$. Define $y_{0}:=\min _{x \in I} f(x)$ and $y_{N}:=\max _{x \in I} f(x)$. Denote the range of $f$ by $R(f):=\left[y_{0}, y_{N}\right]$, where $y_{0} \geqslant 0$ and $y_{N}$ can be infinite which means that $f(x)$ is singular. The foundational equation of NMI proposed by [1] is

$$
(L) \int_{a}^{b} f(x) d x=y_{0} \mu_{f}\left(y_{0}\right)+(R) \int_{y_{0}}^{y_{N}} \mu_{f}(y) d y .
$$

When calculating the Lebesgue integral numerically through (1), there are three kinds of errors [1]:

(i) the error in the method used to estimate $\int_{y_{0}}^{y_{N}} \mu_{f}(y) d y$;

(ii) the error in the estimate of $\mu_{f}(y)$ for some $y$ when necessary;

(iii) the error in estimates of $y_{0}$ and $y_{N}$ whose values are not known.

If the values of $\mu_{f}(y), y_{0}$, and $y_{N}$ can be obtained exactly, the accuracy of the Lebesgue integral of $f(x)$ is determined by the properties of $\mu_{f}(y)$ such as continuity and differentiability. One advantage of this method is that even though $f(x)$ is oscillatory or singular in its bounded domain, its relative measure function $\mu_{f}(y)$ keeps some good properties, bounded and monotonically decreasing. It offers the opportunity to have better results than conventional numerical integral methods.

However, it is often difficult, or perhaps impossible, to find explicit formula for $\mu_{f}(y)$ and exact bound $y_{0}$ and $y_{N}$. The required values need to be estimated which leads to new errors (ii) and (iii) in the numerical results. What we desire is to reduce the effects of the additional errors on the final error of the Lebesgue integration as possible as we can and to obtain the extent of additional errors' effects. Then we need to 
develop methods to estimate the unknown values and analyze the rate of convergence.

In this paper, we will mainly discuss the second kind of error caused by the numerical measures and its effect on the NMI. The paper is organized in 6 sections. In Section 2, we recall a general estimation of the measure function. Then we present its corresponding convergence analysis. Further, by combining Lagrange interpolation, we propose a specific method for approximating the measure function and analyze the rate of convergence. A highly accurate algorithm is presented especially for strictly monotonic functions. In Section 3, we present two examples of estimating measure with one monotonic function and another oscillatory function. In Section 4, we present the error bound of NMI which is controlled by the error of numerical measure and error of integral of exact measure. Further, we prove that the singularity can be reduced by NMI for singular integrals. In Section 5, we give three examples of numerical integrals calculated by NMI based on the numerical measure. Finally, we make a conclusion in Section 6.

\section{Estimation of the Measure Function and Its Convergence Analysis}

To calculate the Lebesgue integrals by the method NMI, it is significant to obtain the values of measure function at some necessary points. However, the values of measure function cannot be obtained exactly through an explicit formula in most of cases. They need to be estimated numerically. And it will be found that the accuracy of the values of measure function will greatly affect the final accuracy of the Lebesgue integral.

To estimate the measure of one-dimensional function $f(x)$, we divide $I$ into $n$ subsets, $\left\{I_{i}:=\left[x_{i}, x_{i+1}\right]: i=\right.$ $1,2, \ldots, n\}$. Define $p_{i}:=m\left(I_{i}\right)=x_{i+1}-x_{i}$ and obviously

$$
\sum_{i=1}^{n} p_{i}=b-a \text {. }
$$

On each interval $I_{i}$, we choose $x_{i} \leqslant x_{i, 1}<x_{i, 2}<\cdots<x_{i, k} \leqslant$ $x_{i+1}, k \in \mathbb{N}$, as testing points. Then the values $\left\{f\left(x_{i j}\right): x_{i j} \in\right.$ $\left.I_{i}, i=1, \ldots, n, j=1, \ldots, k\right\}, k \in \mathbb{N}$, are calculated. Given any $y \in R(f)$, the approximation of $\mu_{f}(y)$ follows

$$
\bar{\mu}_{f}(y)=\sum_{i=1}^{n} \epsilon_{i} p_{i},
$$

where $0 \leqslant \epsilon_{i} \leqslant 1$.

Different methods of choosing $\left\{\epsilon_{i}: i=1,2, \ldots, n\right\}$ will determine different approximations of $\mu_{f}(y)$. A general assignment for $\left\{\epsilon_{i}: i=1,2, \ldots, n\right\}$ which was proposed by [1] is

$$
\epsilon_{i}= \begin{cases}0, & \text { if } y>\max _{1 \leqslant j \leqslant k} f\left(x_{i j}\right), \\ 1, & \text { if } y \leqslant \max _{1 \leqslant j \leqslant k} f\left(x_{i j}\right), \\ \text { an arbitrary number in }(0,1), & \text { otherwise. }\end{cases}
$$

Let $\chi_{E}(x)$ be the characteristic function, defined by

$$
\chi_{E}(x):= \begin{cases}1, & \text { if } x \in E, \\ 0, & \text { if } x \notin E .\end{cases}
$$

Set $g(x):=\chi_{E(f(x) \geqslant y)}(x)$. We have

$$
\mu_{f}(y)=\int_{I} g(x) d x .
$$

Define $\mu_{i}(y):=\int_{I_{i}} g(x) d x$. Then $\mu_{f}(y)=\sum_{i=1}^{n} \mu_{i}(y)$.

Set $M_{y}:=$ number of the segments of $E(f(x) \geqslant y)$. For example, consider $f(x)=|\sin 2 x|$ on $[0, \pi]$; then $M_{y}=2$ for $y \in(0,1)$. The following theorem gives the error analysis of measure approximation (3).

Theorem 1. Let $f(x) \in C[a, b]$ and $f(x) \geqslant 0$ for $x \in[a, b]$. $\left\{I_{i}: i=1, \ldots, n\right\}, n \in \mathbb{N}$, is a partition of $[a, b]$. If $\bar{\mu}_{f}(y)$ and $\left\{\epsilon_{i}: i=1,2, \ldots, n\right\}$ are determined by (3) and (4), respectively, then

$$
\left|\mu_{f}(y)-\bar{\mu}_{f}(y)\right| \leqslant 2 M_{y} \max _{i \in \mathbb{V}} p_{i}
$$

where $\mathbb{V}=\left\{i \in \mathbb{N}: \exists x^{\prime}, x^{\prime \prime} \in I_{i}\right.$ s.t. $\left.f\left(x^{\prime}\right) \leqslant y<f\left(x^{\prime \prime}\right)\right\}$.

Proof. According to the approximate formula (3) and equation (6), we have

$$
\begin{aligned}
\left|\mu_{f}(y)-\bar{\mu}_{f}(y)\right| & =\left|\sum_{i=1}^{n}\left(\mu_{i}(y)-\epsilon_{i} p_{i}\right)\right| \\
& \leqslant \sum_{i=1}^{n}\left|\mu_{i}(y)-\epsilon_{i} p_{i}\right|
\end{aligned}
$$

Set $\mathbb{V}=\left\{i \in \mathbb{N}: \exists x^{\prime}, x^{\prime \prime} \in I_{i}\right.$ s.t. $\left.f\left(x^{\prime}\right) \leqslant y<f\left(x^{\prime \prime}\right)\right\}$. According to the definition of $\epsilon_{i}$, we have $\mu_{i}(y)-\epsilon_{i} p_{i}=0$ for $i \notin \mathbb{V}$. Then the inequality (8) can be simplified as

$$
\left|\mu_{f}(y)-\bar{\mu}_{f}(y)\right| \leqslant \sum_{i \in \mathbb{V}}\left|\mu_{i}(y)-\epsilon_{i} p_{i}\right|
$$

Here it is apparent that

$$
\left|\mu_{i}(y)-\epsilon_{i} p_{i}\right| \leqslant p_{i}
$$

for $0 \leqslant \mu_{i}(y), \epsilon_{i} p_{i} \leqslant p_{i}$.

By the intermediate value theorem, $I_{i}, i \in \mathbb{V}$ contains at least one end of a segment in the domain $E(f(x) \geqslant y)$. Since $E(f(x) \geqslant y)$ has only $M_{y}$ segments, the number of $I_{i}, i \in \mathbb{V}$ is not more than $2 M_{y}$; that is,

$$
\operatorname{Card}(\mathbb{V}) \leqslant 2 M_{y} \text {, }
$$

where Card $(\mathbb{V})$ denotes the cardinality of the set $\mathbb{V}$.

Combining inequalities (9), (10), and (11), we obtain

$$
\left|\mu_{f}(y)-\bar{\mu}_{f}(y)\right| \leqslant 2 M_{y} \max _{i \in \mathbb{V}} p_{i} .
$$


We note that when $M_{y}$ is bounded and the domain $I$ is equally divided into $n$ segments, the numerical measure is uniformly convergent and the order of convergence is linear according to Theorem 1 .

When the integrand functions have some better properties, the numerical measure can be approximated more correctively. Suppose that nonnegative function $f(x)$ is invertible in each subinterval $I_{i}, i=1,2, \ldots, n$. Define $f_{\mathrm{i}}:=$ $\left.f\right|_{I_{i}}$ and $\mu_{f_{i}}:=m\left(E\left(f_{i}(x) \geqslant y\right) \cap I_{i}\right)$. Denote by $R\left(f_{i}\right)$ the range of $f_{i}$ in $I_{i}$ and by $f_{i}^{-1}$ the inverse function of $f_{i}$ in $I_{i}$. Then we obtain

$$
\mu_{f}(y)=\sum_{i=1}^{n} \mu_{f_{i}}(y),
$$

$$
\begin{aligned}
& \mu_{f_{i}}(y) \\
& = \begin{cases}f_{i}^{-1}(y)-x_{i}, & \text { if } f_{i} \text { is decreasing and } y \in R\left(f_{i}\right), \\
x_{i+1}-f_{i}^{-1}(y), & \text { if } f_{i} \text { is increasing and } y \in R\left(f_{i}\right), \\
p_{i}, & \text { if } y<\min _{x \in I_{i}} f_{i}(x), \\
0, & \text { if } y>\max _{x \in I_{i}} f_{i}(x) .\end{cases}
\end{aligned}
$$

Construct a $(k-1)$ th Lagrange interpolation polynomial $P_{i}(x)$ in $R\left(f_{i}\right)$ which interpolates $\left\{\left(f\left(x_{i j}\right), x_{i j}\right): j=\right.$ $1,2, \ldots, k\}$. Then the approximations of $\mu_{f_{i}}(y)$ and $\mu_{f}(y)$ are, respectively,

$$
\begin{aligned}
& \bar{\mu}_{f_{i}}(y) \\
& = \begin{cases}P_{i}(y)-x_{i}, & \text { if } f_{i} \text { is decreasing and } y \in R\left(f_{i}\right), \\
x_{i+1}-P_{i}(y), & \text { if } f_{i} \text { is increasing and } y \in R\left(f_{i}\right), \\
p_{i}, & \text { if } y<\min _{x \in I_{i}} f_{i}(x), \\
0, & \text { if } y>\max _{x \in I_{i}} f_{i}(x), \\
\bar{\mu}_{f}(y)=\sum_{i=1}^{n} \bar{\mu}_{f_{i}}(y) .\end{cases}
\end{aligned}
$$

Set $\epsilon_{i}=\bar{\mu}_{f_{i}}(y) / p_{i}$ which reveals that the estimation (15) is a specific case of the general assignment (4). That means that Theorem 1 is valid for the estimation (15).

Theorem 2. Suppose that nonnegative function $f(x)$ has a partition $\left\{I_{i}: i=1,2, \ldots, n\right\}$ in $I$ and has inverse function $f_{i}^{-1} \in C^{k}\left(R\left(f_{i}\right)\right)$ in each subinterval. If $\bar{\mu}_{f}(y)$ are determined by (14) and (15), then

$$
\left\|\mu_{f}(y)-\bar{\mu}_{f}(y)\right\|_{\infty}=\mathcal{O}\left(n^{-k}\right)
$$

where $\mathbb{V}=\left\{i \in \mathbb{N}: \exists x^{\prime}, x^{\prime \prime} \in I_{i}\right.$ s.t. $\left.f\left(x^{\prime}\right) \leqslant y<f\left(x^{\prime \prime}\right)\right\}$.
Proof. According to Theorem 1 and (13), (14), and (15), we have

$$
\begin{aligned}
& \left|\mu_{f}(y)-\bar{\mu}_{f}(y)\right| \\
& \quad \leqslant \min \left(n \max _{i}\left\|f_{i}^{-1}(y)-P_{i}(y)\right\|_{\infty}, 2 M_{y} \max _{i \in \mathbb{V}} p_{i}\right) .
\end{aligned}
$$

The proof is finished combining the well-known error of Lagrange interpolation polynomials.

According to the proof of Theorem 2, the accuracy of measure function depends on the accuracy of Lagrange interpolation polynomials of the inverse functions in each subinterval. Numerical experiments will be done to affirm the validity of the method. At the end of this section, we would like to introduce a highly accurate algorithm to calculate the measure especially for strictly monotonic functions. It is called split-half algorithm.

Algorithm 3 (split-half algorithm). Consider the following steps.

Step 1. Given $y \in R(f)$ and $N \in \mathbb{N}$, set $i:=0, \Delta=b-a$, $x_{m}=(b-a) / 2$, and $\mu_{f}^{i}(y)=0$. Determine the monotonicity of the function $f$, increasing or decreasing.

Step 2. Evaluate the function at $x_{m}$ :

$$
T_{1}:= \begin{cases}1, & f\left(x_{m}\right)>y, \\ -1, & f\left(x_{m}\right)<y, \\ 0, & f\left(x_{m}\right)=y .\end{cases}
$$

Step 3. If $T_{1}=0$, we can get the accurate measure

$$
\mu_{f}^{i+1}(y)=\mu_{f}^{i}(y)+\frac{\Delta}{2} .
$$

Otherwise,

$$
\mu_{f}^{i+1}(y)=\mu_{f}^{i}(y)+\frac{T_{1}+1}{2} \frac{\Delta}{2} .
$$

Set $i \leftarrow i+1, \Delta \leftarrow \Delta / 2$, and $x_{m} \leftarrow x_{m}-T_{1} \Delta / 2$ for increasing functions or $x_{m} \leftarrow x_{m}+T_{1} \Delta / 2$ for decreasing functions. And go back to Step 2 until $i=N$.

The error of split-half algorithm satisfies

$$
\left\|\mu_{f}(y)-\mu_{f}^{N}(y)\right\|_{\infty} \leqslant 2^{-N}(b-a) .
$$

\section{Numerical Examples of Measure}

Example 1. Consider $f(x)=\ln x, x \in[1,5]$.

Since $\ln x$ is monotonically increasing, $y_{0}=0, y_{N}=\ln 5$, and $\mu_{f}(y)=5-e^{y}, y \in R(f) . f^{-1}(x) \in C^{\infty}(R(f))$.

Table 1 lists the respective measure errors and their convergence orders (briefly, C.O.). The error of the approximation for values $n$ and $k$ is presented by $e_{n, k}:=$ $\left\|\mu_{f}(y)-\bar{\mu}_{f}(y)\right\|_{\infty}$, where $\bar{\mu}_{f}(y)$ is determined by (14) and each convergence order is computed by $\ln \left(e_{n} / e_{m}\right) / \ln (m / n)$. 
TABLE 1: Errors of $\mu(y)$.

\begin{tabular}{lcccc}
\hline$n$ & Errors $(k=2)$ & C.O. & Errors $(k=4)$ & C.O. \\
\hline 8 & $2.5284 e-002$ & & $2.2050 e-005$ & \\
16 & $6.9679 e-003$ & 1.8594 & $1.6494 e-006$ & 3.7408 \\
32 & $1.8272 e-003$ & 1.9311 & $1.1349 e-007$ & 3.8614 \\
64 & $4.7207 e-004$ & 1.9526 & $5.4007 e-009$ & 4.3932 \\
128 & $1.1997 e-004$ & 1.9763 & $3.9920 e-010$ & 3.7580 \\
256 & $2.7692 e-005$ & 2.1151 & $2.6089 e-011$ & 3.9356 \\
512 & $7.1502 e-006$ & 1.9534 & $1.5805 e-012$ & 4.0450 \\
\hline
\end{tabular}

TABLE 2: Errors of $\mu(y)$.

\begin{tabular}{lcccc}
\hline$n$ & Errors $(k=2)$ & C.O. & Errors $(k=4)$ & C.O. \\
\hline 20 & $2.2045 e-001$ & & $1.0791 e-001$ & \\
40 & $1.2578 e-001$ & .80948 & $8.3284 e-002$ & .37371 \\
80 & $6.4771 e-002$ & .95754 & $4.6044 e-002$ & .85501 \\
160 & $2.6235 e-002$ & 1.3039 & $6.8435 e-003$ & 2.7502 \\
320 & $5.3436 e-003$ & 2.2956 & $7.4735 e-005$ & 6.5168 \\
640 & $6.7018 e-004$ & 2.9952 & $1.7822 e-005$ & 2.0682 \\
1280 & $2.6427 e-004$ & 1.3425 & $4.8946 e-007$ & 5.1863 \\
\hline
\end{tabular}

Example 2. Consider an oscillatory function $f(x)=$ $|\sin 30 x|, x \in[0, \pi / 3]$.

The minimum and maximum of $f(x)$ are $y_{0}=0$ and $y_{N}=$ 1 , respectively. For $f(x)$ is a periodic function, we can obtain the explicit formula of measure function:

$$
\mu(y)=\frac{1}{3}(\pi-2 \arcsin y), \quad y \in R(f) .
$$

Numerical results of errors are shown in Table 2. Since the measure function does not possess good differentiability when $y=0$, the errors do not have stable convergence order of $\mathcal{O}\left(n^{-k}\right)$.

\section{Error Analysis of NMI}

In this section, we will analyze the whole error of the integral $\int_{a}^{b} f(x) d x$. As we care greatly about the influence of errors caused by the numerical measures, we assume that the maximum and minimum of the function $f(x)$ are known; that is, we will not consider the errors of $y_{0}$ and $y_{N}$ here. To analyze the error of the integral $\int_{a}^{b} f(x) d x$, it is necessary to choose an integral rule for the Riemann integral of the measure function. An appropriate rule for the onedimensional integral is dependent on the character of $\mu(y)$.

Let $U \subset \mathbb{R}$ be an interval and assume $g \in C(U)$. To approximate the integral $Q(g):=\int_{U} g(t) d t$, we consider numerical quadrature rule of the form

$$
Q_{n}(g):=\sum_{j=0}^{n} w_{n, j} g\left(t_{n, j}\right),
$$

where $t_{n, j} \in U, j=0,1, \ldots, n$, are quadrature points and $w_{n, j}, j=0,1, \ldots, n$, are real quadrature weights.
Define $E_{Q_{n}}(g):=Q(g)-Q_{n}(g)$ the integral error of $g$ under the given quadrature rule.

Integrating the measure function by the numerical quadrature rule (23) in the fundamental equation (1), we have

$$
\begin{aligned}
& \int_{I} f(x) d x \\
& \quad=y_{0}(b-a)+Q_{n}\left(\mu_{f}\right)+E_{Q_{n}}\left(\mu_{f}\right) \\
& \quad=y_{0}(b-a)+Q_{n}\left(\bar{\mu}_{f}\right)+Q_{n}\left(\mu_{f}-\bar{\mu}_{f}\right)+E_{Q_{n}}\left(\mu_{f}\right),
\end{aligned}
$$

where $\mu_{f}$ and $\bar{\mu}_{f}$ are, respectively, the measure function and numerical measure function of $f(x)$. So the numerical quadrature rule of NMI corresponding to $f$ is

$$
L Q_{n}(f):=y_{0}(b-a)+Q_{n}\left(\bar{\mu}_{f}\right)
$$

Define $E_{L Q_{n}}(f):=\int_{I} f(x) d x-L Q_{n}(f)$. We have the following fundamental theorem for the error of NMI.

Theorem 4. Let $f(x) \in \mathbb{C}[a, b]$ and $f(x) \geqslant 0$ for $x \in[a, b]$. By estimating $\int_{I} f(x) d x$ by the numerical quadrature rule of NMI (25), the error satisfies

$$
\begin{aligned}
\left|E_{L Q_{n}}(f)\right| & \leqslant\left|Q_{n}\left(\mu_{f}-\bar{\mu}_{f}\right)\right|+\left|E_{Q_{n}}\left(\mu_{f}\right)\right| \\
& \leqslant\left\|\mu_{f}-\bar{\mu}_{f}\right\|_{\infty} \sum_{j=0}^{n}\left|w_{n, j}\right|+\left|E_{Q_{n}}\left(\mu_{f}\right)\right| .
\end{aligned}
$$

According to Theorem 4, the accuracy of numerical quadrature rule of NMI depends on the accuracy of numerical measure and the character of measure function $\mu_{f}$. A necessary condition to have better accuracy by NMI is that the measure function $\mu_{f}$ should possess better properties. For bounded oscillatory functions, the integral of the exact measure function can be approximated more accurate for its monotonic property. As to singular functions, it can be verified in the following that NMI can decrease the degree of the singularity of the integral.

When $y_{N}$ is infinite, by making the variable change of $z=1 / y$ in the fundamental equation (1) of NMI, it can be rewritten as

$$
\int_{I} f(x) d x=y_{0}(b-a)+\int_{0}^{1 / y_{0}} \frac{1}{z^{2}} \mu_{f}\left(\frac{1}{z}\right) d z
$$

where $y_{0}$ is supposed to be $y_{0} \geqslant \epsilon>0$.

Denote $g(x):=\left(1 / x^{2}\right) \mu_{f}(1 / x), x \in\left(0,1 / y_{0}\right]$. Before we prove that $g(x)$ is less singular than $f(x)$, a lemma should be stated.

Lemma 5. Let $f(x) \in C(I), h(t):=f(x(t))$, and $x(t):=k t+c$ where $k>0, c \in \mathbb{R}$. $\mu_{f}$ and $\mu_{h}$ are the measure functions corresponding to $f$ and $h$, respectively. Then $\mu_{f}(y)=k \mu_{h}(y)$, $\forall y \in R(f)$, where $R(f)$ is the range of $f$. 
Proof. Since $k>0, g$ has the same monotonicity as $f$. When $f$ is monotonically decreasing, $\mu_{f}(y)=f^{-1}(y)-a$ and $\mu_{h}(y)=h^{-1}(y)-(a-c) / k$, respectively. We obtain

$$
h^{-1}(y)=\frac{1}{k}\left(f^{-1}(y)-c\right) .
$$

Substituting the inverse function of $h$ into $\mu_{h}$, we have

$$
\mu_{h}(y)=\frac{1}{k}\left(f^{-1}(y)-a\right)=\frac{\mu_{f}(y)}{k} .
$$

When $f$ is monotonically increasing, $\mu_{f}(y)=b-f^{-1}(y)$ and $\mu_{h}(y)=(b-c) / k-h^{-1}(y)$, respectively. It can be obtained similarly that $\mu_{h}(y)=\mu_{f}(y) / k$.

For any $f \in C(I)$, there is a partition $\left\{I_{i}: i \in \mathbb{N}\right\}$ of $I$ such that $f$ is monotonic in each subinterval. Define $f_{i}:=\left.f\right|_{I_{i}}$ and $\mu_{f_{i}}:=m\left(E\left(f_{i} \geqslant y\right) \cap I_{i}\right), i \in \mathbb{N}$. Correspondingly, define $h_{i}:=f_{i}(x(t))$ and $\mu_{h_{i}}:=m\left(E\left(h_{i} \geqslant y\right) \cap x^{-1}\left(I_{i}\right)\right), i \in \mathbb{N}$.

Then it is obvious that

$$
\mu_{f}(y)=\sum_{i \in \mathbb{N}} \mu_{f_{i}}(y), \quad \mu_{h}(y)=\sum_{i \in \mathbb{N}} \mu_{h_{i}}(y) .
$$

According to the preceding proof,

$$
\mu_{f_{i}}(y)=k \mu_{h_{i}}(y)
$$

since $f_{i}, \forall i \in \mathbb{N}$, is monotonic in its domain. Combining (30) and (31), we finally arrive at the equation $\mu_{f}(y)=k \mu_{h}(y)$.

Let $S$ be a set of $I=[a, b]$ containing a finite number of points. Define a function associated with $S$ by

$$
w_{S}:=\inf \{|x-t|: t \in S\} .
$$

For $0<\alpha<1$, a real unbounded function $f$ is said to be of Type $(\alpha, I, S)$, if

$$
|f(x)| \leqslant C\left[w_{S}(x)\right]^{-\alpha}, \quad x \notin S, f \in C(I \backslash S),
$$

where $C$ is a positive constant. The parameter $\alpha$ is called index of singularity.

Theorem 6. Let $f \in T y p e(\alpha, I, S)$ and $f(x) \geqslant \epsilon>0$ for $x \in I$. $g(x)=\left(1 / x^{2}\right) \mu_{f}(1 / x), x \in\left(0,1 / y_{0}\right]$, where $\mu_{f}$ is the measure function of $f$ and $y_{0}=\min _{x \in I} f(x) \geqslant \epsilon>0$. Then $g(x) \epsilon$ Type $\left((2 \alpha-1) / \alpha,\left(0,1 / y_{0}\right],\{0\}\right)$.

Proof. Assume $S=\{a\}$ or $\{b\}$. By making the variable change $x(t)=(b-a) t+a$ for $S=\{a\}$ or $x(t)=(b-a) t+b$ for $S=\{b\}$, we define $h(t):=f(x(t)), t \in(0,1]$. Then $h \in$ Type $(\alpha,(0,1],\{0\})$.

Since $h(t)$ is singular at $t=0$, there exists $t_{0} \in(0,1]$ such that $h(t)$ is monotonic in the interval $\left(0, t_{0}\right]$. That means that $\forall y \geqslant h^{-1}\left(t_{0}\right)$ s.t. $\mu_{h}(y)=\left|h^{-1}(y)\right|$. According to the property of $h$, we can obtain

$$
y=h\left(h^{-1}(y)\right) \leqslant \frac{1}{\left|h^{-1}(y)\right|^{\alpha}} .
$$

The resultant inequality reveals that $\mu_{h}(y) \leqslant y^{-1 / \alpha}$.
By Lemma 5, we have $\mu_{f}(y)=(b-a) \mu_{h}(y) \leqslant(b-a) y^{-1 / \alpha}$.

Then combining the inequality of $\mu_{f}$ and the expression of $g$, we can obtain $0 \leqslant g(x) \leqslant(b-a) x^{-(2 \alpha-1) / \alpha}, \forall x \in\left(0, t_{0}\right]$.

Since $g(x)$ is continuous in $\left[t_{0}, 1 / y_{0}\right]$, there exists a positive constant $C$ such that $\forall x \in\left(0,1 / y_{0}\right]$,

$$
0 \leqslant g(x) \leqslant C x^{-(2 \alpha-1) / \alpha} .
$$

According to the definition (33), we have $g(x) \in$ Type $((2 \alpha-$ $\left.1) / \alpha,\left(0,1 / y_{0}\right],\{0\}\right)$.

More generally, $S$ contains more than one point. Assume $S=\left\{a=s_{1}<s_{2}<\cdots<s_{m}=b\right\}$. Set

$$
\begin{gathered}
t_{2 i}=s_{i}, \quad i=1,2, \ldots, m \\
t_{2 i-1}=\frac{1}{2}\left(s_{i}+s_{i+1}\right), \quad i=1,2, \ldots, m-1 .
\end{gathered}
$$

In each interval $\left(t_{i}, t_{i+1}\right)$, the function $f(x)$ has only one singularity. Namely, a singularity is located at $t_{i}$ if $i$ is even and at $t_{i+1}$ if $i$ is odd.

Define $f_{i}(x):=\left.f(x)\right|_{\left(t_{i}, t_{i+1}\right)}, \mu_{f_{i}}(y):=m(E(f(x) \geqslant y) \cap$ $\left.\left(t_{i}, t_{i+1}\right)\right)$, and $g_{i}(y):=\left(1 / y^{2}\right) \mu_{f_{i}}(1 / y)$. Then we have

$$
\mu_{f}(y)=\sum_{i=1}^{2 m} \mu_{f_{i}}(y), \quad g(y)=\sum_{i=1}^{2 m} g_{i}(y) .
$$

By the preceding proof, it can be obtained that $0 \leqslant g_{i}(x)<$ $C_{i} x^{-(2 \alpha-1) / \alpha}, \forall x \in\left(0,1 / y_{0 i}\right]$, where $y_{0 i}:=\min _{x \in\left(t_{i}, t_{i+1}\right)} f(x) \geqslant$ $y_{0}$ and $C_{i}$ is a positive constant, $i=1,2, \ldots, 2 \mathrm{~m}$. Since $\mu_{f_{i}}(1 / x)=0$ for $x \in\left(1 / y_{0 i}, 1 / y_{0}\right], 0 \leqslant g_{i}(x)<C_{i} x^{-(2 \alpha-1) / \alpha}$ is valid for $x \in\left(0,1 / y_{0}\right]$. Combining (37), we prove that $g(x) \in$ Type $\left((2 \alpha-1) / \alpha,\left(0,1 / y_{0}\right],\{0\}\right)$.

Since $(2 \alpha-1) / \alpha<\alpha, \forall \alpha \in(0,1)$, NMI can reduce the singularity for singular integrals. It supplies the possibility to improve the accuracy of integrals. Theoretically, we need not know where the singularities lie.

As to whether NMI can realize the better accuracy of integrals, it depends on the accuracy of the numerical measure. In the following part, we will present some numerical integrals results by NMI and compare them with results by conventional methods.

\section{Numerical Examples of NMI}

In this section, we will present three kinds of integrals, including normal, oscillatory, and singular integrals, to verify the efficiency of NMI. In the numerical experiments, the Gauss-Legendre formula or its composite one will be used to calculate the integral of measure functions.

Example 1. Consider the integration $\int_{1}^{5} \ln (x) d x=5 \ln 5-4$.

We have known that $y_{0}=0, y_{N}=\ln 5$, and $\mu(y)=5-e^{y}$, $y_{0} \leqslant y \leqslant y_{N}$.

In this numerical example, we adopt 5-point GaussLegendre formula to calculate the integral of measure function and original integral. The errors of original integral and 
TABLE 3: Errors of integration $\int_{1}^{5} \ln (x) d x$ by NMI.

\begin{tabular}{llll}
\hline$n$ & Errors $(k=1)$ & Errors $(k=3)$ & Errors $(k=5)$ \\
\hline 8 & $3.0958 e-001$ & $1.9666 e-005$ & $3.4806 e-008$ \\
16 & $9.9805 e-002$ & $1.7423 e-005$ & $2.5702 e-009$ \\
32 & $7.5973 e-002$ & $4.3251 e-006$ & $2.3129 e-010$ \\
64 & $6.4057 e-002$ & $5.6059 e-007$ & $1.6858 e-010$ \\
128 & $2.8068 e-002$ & $2.2432 e-008$ & $1.6776 e-010$ \\
256 & $1.3053 e-002$ & $3.5017 e-009$ & $1.6779 e-010$ \\
512 & $7.9865 e-003$ & $5.2533 e-010$ & $1.6778 e-010$ \\
\hline
\end{tabular}

TABLE 4: Errors of integration $\int_{0}^{\pi / 3}|\sin 30 x| d x$.

\begin{tabular}{lcc}
\hline$n$ & $\mathrm{CS}$ & $\mathrm{GL}$ \\
\hline 8 & $1.5199 e-003$ & $5.0402 e-004$ \\
16 & $8.9723 e-005$ & $1.1475 e-005$ \\
32 & $5.5303 e-006$ & $9.1145 e-008$ \\
64 & $3.4446 e-007$ & $4.8650 e-010$ \\
128 & $2.1510 e-008$ & $2.1969 e-012$ \\
256 & $1.3441 e-009$ & $8.9928 e-015$ \\
512 & $8.4001 e-011$ & $1.1102 e-016$ \\
\hline
\end{tabular}

the integral of exact measure function are 3.4407e - 005 and $1.6778 e-010$, respectively. It shows clearly that the accuracy of the numerical integral can be greatly improved by making the NMI transform for this example. Numerical results of errors of the integration by NMI are shown in Table 3. The measure is estimated by (14) and (15) with values $n$ and $k$. The high accuracy of numerical results of NMI requires the accuracy of measure functions as illustrated in Table 3.

Example 2. Consider the integration of an oscillatory function $\int_{0}^{\pi / 3}|\sin 30 x| d x=2 / 3$.

In this example, $y_{0}=0, y_{N}=1$, and $\mu(y)=\pi / 3-$ $(2 \arcsin (y) / 3), y_{0} \leqslant y \leqslant y_{N}$. Since the measure function is weakly singular at $y=1$, we adopt the Gauss-type quadrature for weakly singular integrals proposed by [2]. According to the quadrature rule, we set the parameters $k=4$ and $q=6$. In detail, set $N=n / k$, where $n$ is the number of evaluations of the function showed in Table 4 . Then choose $(N+1)$ points $t_{j}=1-(j / N)^{q}$ so that the subintervals $I_{j}:=\left[t_{j}, t_{j+1}\right], j=$ $0,1, \ldots, N$, form a partition for $[0,1]$. Then transform the partition into the domain of integral and use k-point GaussLegendre formula in each subinterval to calculate the integral. By the quadrature, the errors of the integral with exact measure function is showed in Table 4 represented by GL. To show the efficiency of the proposed method, we compare the results with those obtained by composite Simpson's rule with $n+1$ evaluations of function which is denoted by CS in Table 4 . It is obvious that the accuracy can be improved under the correct measure function.

Errors of the integration by NMI combining GL are shown in Table 5 which is denoted by GLNMI. We set the number of evaluations of function 32 for the integral of measure function by the Gauss-type quadrature. The measure
TABLE 5: Errors of integration $\int_{0}^{\pi / 3}|\sin 30 x| d x$ by GLNMI.

\begin{tabular}{llll}
\hline$n$ & Errors $(k=1)$ & Errors $(k=3)$ & Errors $(k=5)$ \\
\hline 20 & $1.4307 e-001$ & $3.1465 e-002$ & $2.7730 e-002$ \\
40 & $4.5358 e-002$ & $7.8500 e-003$ & $1.1460 e-002$ \\
80 & $3.9992 e-003$ & $1.2441 e-003$ & $1.8890 e-003$ \\
160 & $1.5046 e-002$ & $1.4846 e-004$ & $1.8805 e-004$ \\
320 & $3.9658 e-003$ & $2.6296 e-005$ & $3.4863 e-005$ \\
640 & $4.1803 e-003$ & $2.1141 e-006$ & $7.1482 e-006$ \\
1280 & $2.9805 e-004$ & $4.0182 e-007$ & $2.3580 e-007$ \\
\hline
\end{tabular}

TABLE 6: Errors of integration $\int_{0}^{1}\left(1 / x^{0.7}\right) d x$.

\begin{tabular}{lcc}
\hline$n$ & GL & GLNMI \\
\hline 8 & $7.5026 e-001$ & $2.1705 e-001$ \\
16 & $2.1096 e-001$ & $3.9276 e-002$ \\
32 & $2.7858 e-002$ & $4.0278 e-003$ \\
64 & $2.4575 e-003$ & $3.1686 e-004$ \\
128 & $1.8065 e-004$ & $2.2109 e-005$ \\
256 & $1.2212 e-005$ & $1.4597 e-006$ \\
512 & $7.9320 e-007$ & $9.5708 e-008$ \\
\hline
\end{tabular}

is estimated by (14) and (15) with values $n$ and $k$. The accuracy of NMI is extremely restricted by the accuracy of measure.

Example 3. Consider the integration of a singular function $\int_{0}^{1}\left(1 / x^{0.7}\right) d x=10 / 3$.

Since the integral and the measure integral are both weakly singular, we adopt the Gauss-type quadrature used in Example 2. We set the parameters $k=2$ and $q=50 / 3$. Splithalf algorithm is used to approximate the measure function with $N=100$ in this example. The numerical results are presented in Table 6 where GL represents using only the method of Gauss-type quadrature while GLNMI represents combining NMI and Gauss-type quadrature together. It is illustrated that the accuracy can be improved by NMI.

\section{Conclusion}

In this paper, we mainly discuss how to estimate the measure of a function and the convergence of the numerical measure. Further, we analyze the error of numerical measure integral and verify that the singular integrals can be improved theoretically. Accordingly, some numerical examples are given to validate the theoretical results.

To calculate the integration by NMI, it is greatly important to estimate the measures accurately as possible as we can. The method presented here is very effective for functions which have piecewise differential inverse function.

The study of the NMI field is still new. Investigations should be expanded to the multidimensional measures and integrations. Maybe, the method NMI is more suitable for multidimensionality because it presents an exact reduction of multidimensional integrals to one-dimensional integrals. More researches can be done in this field. 


\section{Acknowledgment}

The authors are very grateful to Professor Tian-xiao He for his kind help and valuable recommendations. This work is partially supported by National Natural Science Foundation of China under Projects 11271370 and 61101183 and CSC Scholarship.

\section{References}

[1] B. L. Burrows, "A new approach to numerical integration," Journal of the Institute of Mathematics and Its Applications, vol. 26, no. 2, pp. 151-173, 1980.

[2] H. Kaneko and Y. Xu, "Gauss-type quadratures for weakly singular integrals and their application to Fredholm integral equations of the second kind," Mathematics of Computation, vol. 62, no. 206, pp. 739-753, 1994. 


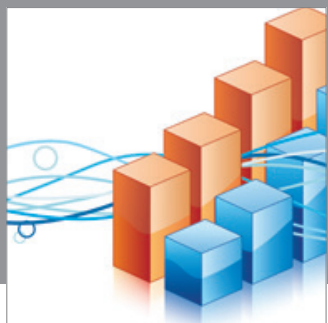

Advances in

Operations Research

mansans

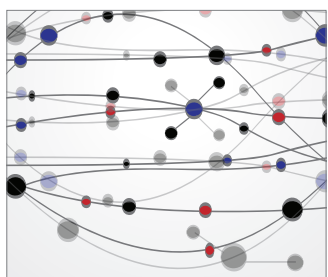

The Scientific World Journal
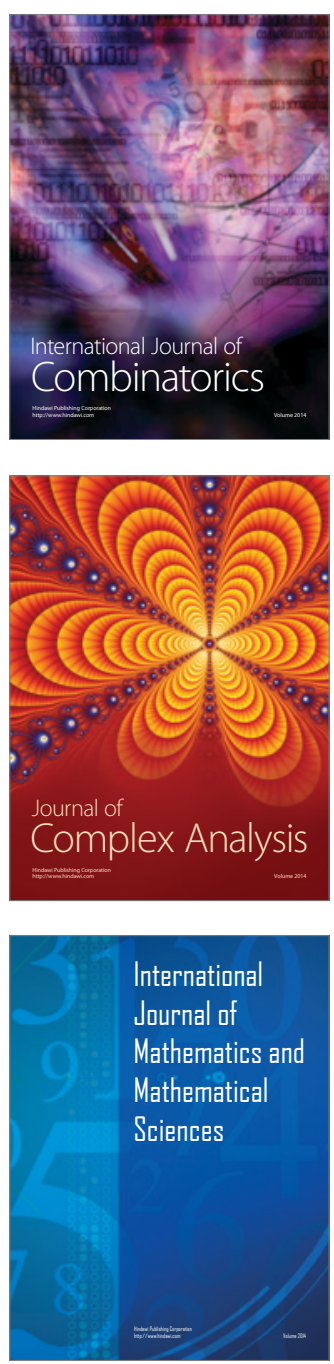
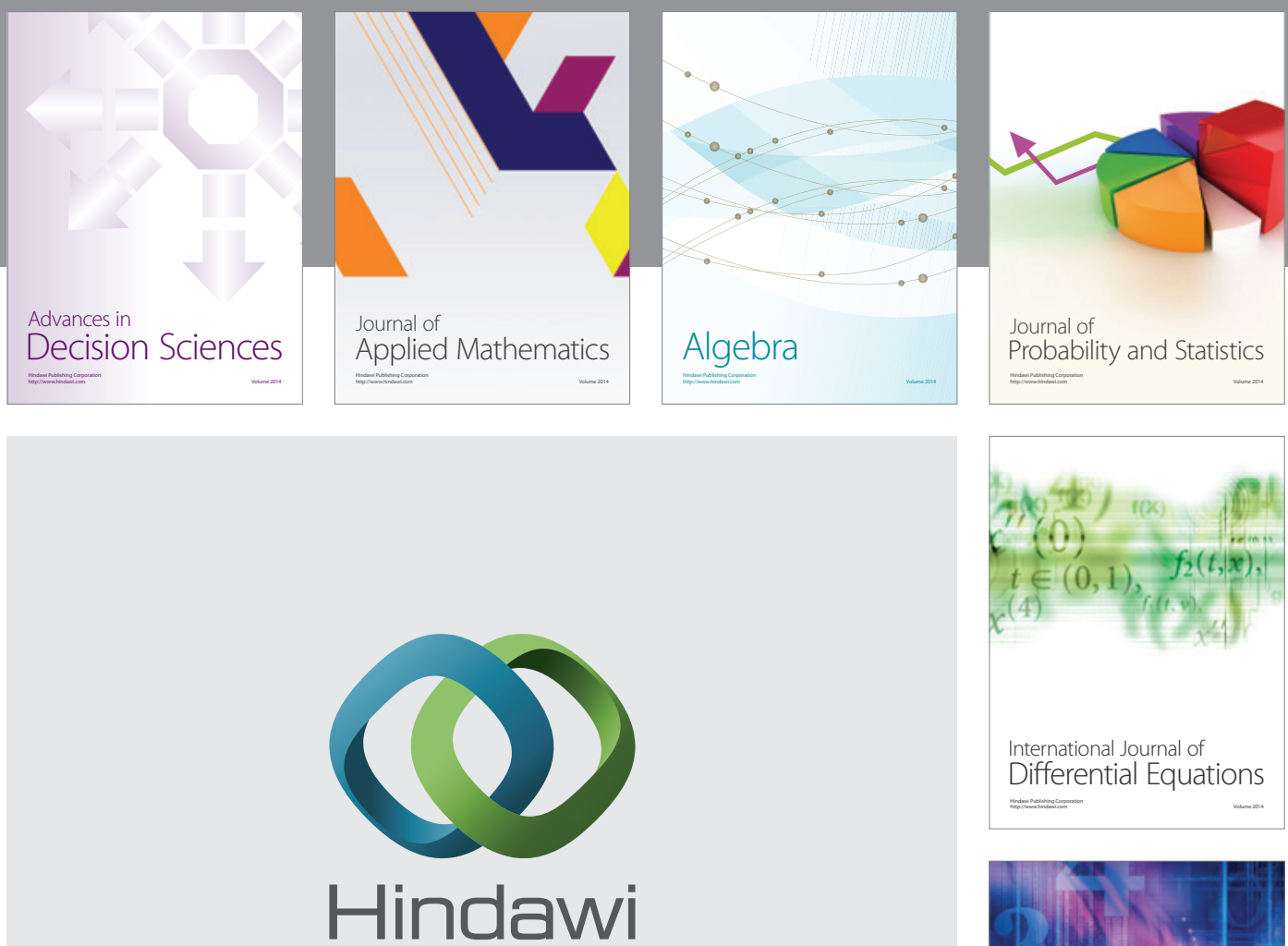

Submit your manuscripts at http://www.hindawi.com
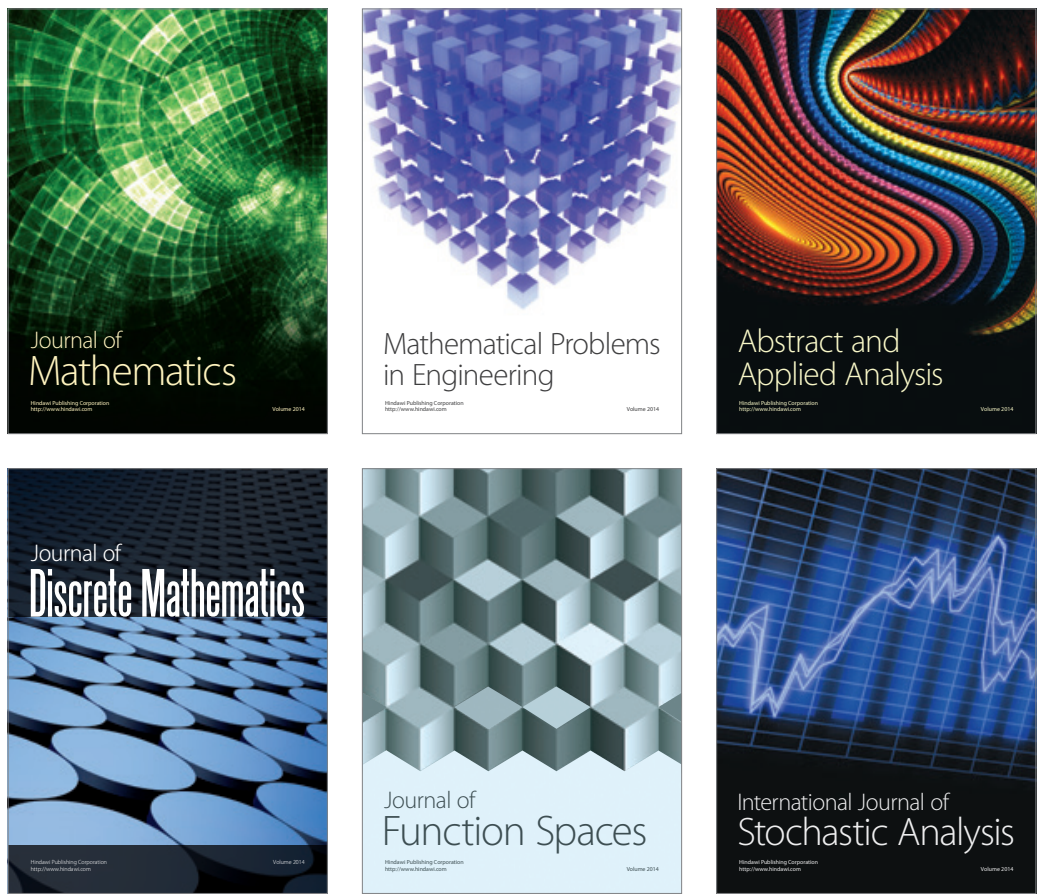

Journal of

Function Spaces

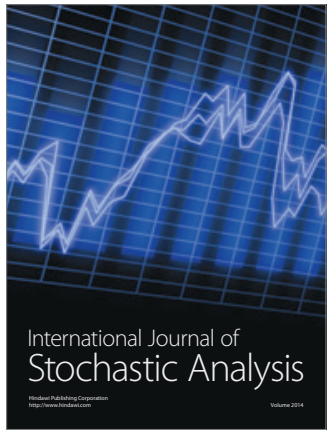

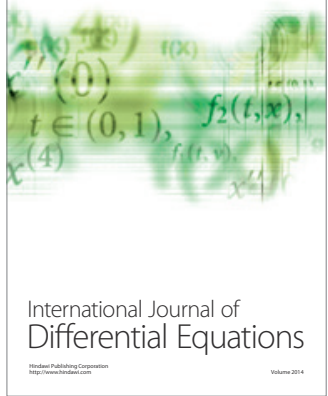
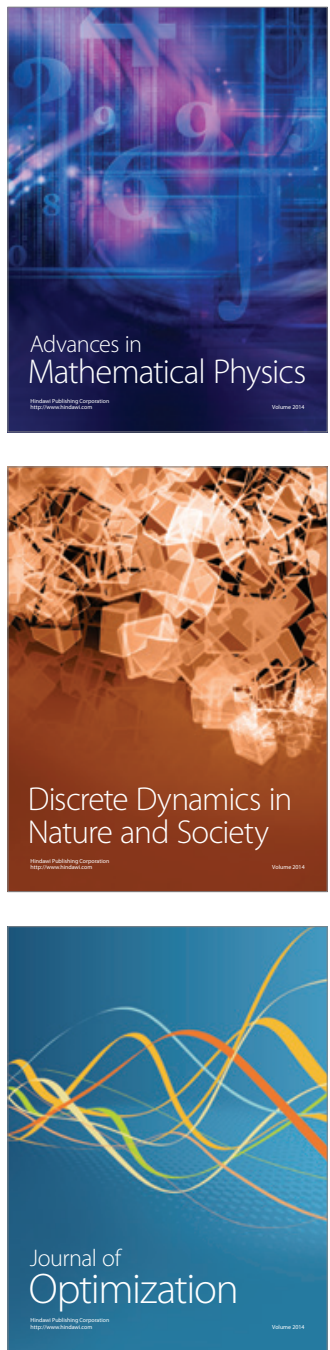